\title{
pth moment stability in stochastic neutral Volterra-Levin equation with Lévy noise and variable delays
}

\author{
Hongwei Yin, Shuiming Xiao*, Xiaoyong Xiao and Xiaoqing Wen
}

"Correspondence:

xsm9723009@163.com School of Science, Nanchang Univeristy, Xufu Big Street, No 999, Nanchang, P.R. China

\begin{abstract}
In this paper, we study a class of stochastic neutral Volterra-Levin equations which are equipped with Lévy noise and variable delay and we obtain pth moment exponential stability. Some well-known results are improved and generalized.
\end{abstract}

Keywords: fixed point theory; Lévy noise; neutral differential equation; variable delay; $p$ th moment exponential stability

\section{Introduction}

Plenty of sophisticated dynamical systems are described by stochastic differential equations in physics, biology, chemistry, economics, control engineering, and financial market theory [1]. Now, first order nonlinear stochastic equations have recently received a lot of attention on the investigation of stability in the literature; for instance, see the book [2] and specially important papers $[3,4]$. Moreover, the asymptotic stability results for various second-order stochastic differential equations have also been established in [5-7]. It is well known that Lyapunov's method has been the classical technique to study stability of deterministic and stochastic differential equations and functional differential equations for more than 100 years, for example [1, 2]. However, there are a lot of difficulties to construct Lyapunov functions for examining stability. Burton et al. [8-11] have successfully applied fixed point theory to overcome these problems.

Volterra-Levin equations are often applied in biological problem [12], which were first studied by Volterra. Specially, stability of Volterra-Levin equation always attracts many authors' attentions. In detail, Zhao and Yuan in [13] obtained 3/2 stability conditions for determinate Volterra-Levin equation. Furthermore, by fixed point theory, Lou in [14] studied the stochastic Voltera-Levin equation which is equipped with constant delay and Gaussian noise, and he obtained the mean square exponential stability. Unfortunately, the stochastic disturbances in real world do not obey Gaussian noise, but they are variable structures subject to stochastic abrupt changes, which may come from abrupt phenomena. For this case, it is recognized that stochastic differential equations with Lévy noise are quite suitable to describe such stochastic abrupt phenomena. Thus, Guo and Zhu in [15] studied the stochastic Voltera-Levin equation with Poisson jumps, which is a special case of Lévy noise, and they obtained $p$ th moment stability of this equations. Their results are more generalized and obviously extended ones in [16]. Real systems depend on not only present

(2014 Yin et al: licensee Springer. This is an Open Access article distributed under the terms of the Creative Commons Attribution License (http://creativecommons.org/licenses/by/2.0), which permits unrestricted use, distribution, and reproduction in any medium, provided the original work is properly cited. 
and past states but also on derivatives with delays. As a result, these systems are often built in the form of neutral differential equations. Recently, Ardjouni and Djoudi in [17] have addressed the deterministic neutral Volterra-Levin equation with variable delay by using fixed point theory. As far as we know, the stochastic version for a neutral Volterra-Levin equation with variable delays and Lévy noise has been not investigated. To this end, in this paper we make the first attempt to fill this gap and study $p$ th moment exponential stability of the stochastic neutral Volterra-Levin equation with variable delays and Lévy noise by fixed point theory and using the Bukhölder-Davis-Gundy inequality.

This paper is organized as follows. In Section 2, some necessary basic preliminaries and the form of the stochastic neutral Volterra-Levin equation with variable delays and Lévy noise are introduced. In Section 3, we show the main result about $p$ th moment exponential stability and the proof of this result. In Section 4, we make some remarks and an example is given to test our result. Finally, a necessary discussion is given.

\section{Preliminaries}

Let $\left\{\Omega, \mathcal{F},\{\mathcal{F}\}_{t \geq 0}, \mathbf{P}\right\}$ be a probability space with a filtration satisfying the usual conditions, i.e., the filtration is continuous on the right and $\mathcal{F}_{0}$ contains all $\mathbf{P}$-zero sets. Let $\{B(t), t \geq 0\}$ be a standard Brownian motion defined on $\{\Omega, \mathcal{F}, \mathbf{P}\} . \tilde{N}(d t, d u), t \in R^{+}, u \in R$ is a centered Poisson random measure with parameter $\pi(d u) d t$.

Now, we consider the following stochastic neutral Volterra-Levin equation with variable delay and Lévy noise:

$$
\begin{aligned}
d\left[x(t)-G\left(t, x\left(t-\tau_{1}(t)\right)\right)\right]= & -\left(\int_{t-\tau_{2}(t)}^{t} a(t, s) g(x(s)) d s\right) d t \\
& +\sigma\left(t, x(t), x\left(t-\tau_{3}(t)\right)\right) d B(t) \\
& +\int_{R} h\left(t, x(t), x\left(t-\tau_{4}(t)\right), u\right) \tilde{N}(d t, d u), \quad t \geq 0,
\end{aligned}
$$

with the initial condition $x_{0}=\psi(t)$, where $\psi(t) \in C([-m(0), 0] ; R)$ and $-m(0)=\min _{s \geq 0}\{s-$ $\left.\tau_{i}(s), i=1,2,3,4\right\}$.

Assume that the function $G\left(t, x\left(t-\tau_{1}(t)\right)\right)$ is continuous and $G(t, 0)=g(0)=\sigma(0,0,0)=$ $h(0,0,0, u)=0, \tau_{i}(t) \in C\left(R^{+}, R^{+}\right)$satisfies $t-\tau_{i}(t) \rightarrow \infty, i=1,2,3,4 . \tilde{N}(d t, d u)=N(d t, d u)-$ $\pi(d u) d t$ is a compensated Poisson random measure which is independent of $\{B(t)\}_{t \geq 0}$.

We assume that the following conditions hold:

(i) there exists a positive constant $k_{1}$ such that $|G(t, x)-G(t, y)| \leq k_{1}|x-y|$;

(ii) there exists a positive $k_{2}$ such that

$$
|a(u, s)| \leq k_{2}, \quad \text { for } u \in[-m(0), \infty), s \in R^{+} ;
$$

(iii) there exists a positive $k_{3}$ such that

$$
|g(x)-g(y)| \leq k_{3}|x-y|
$$

(iv) $\tau_{2}(t)$ is differential with respect to $t$ and there exists a positive constant $k_{4}$ such that $\left|\tau_{2}^{\prime}(t)\right| \leq k_{4}, t \in R^{+}$; 
(v) the global Lipschitz condition: there exists a positive constant $k_{5}$ such that

$$
\begin{aligned}
& \left|\sigma\left(t, x_{1}, y_{1}\right)-\sigma\left(t, x_{2}, y_{2}\right)\right|^{2} \vee \int_{R}\left|h\left(t, x_{1}, y_{1}, u\right)-h\left(t, x_{2}, y_{2}, u\right)\right|^{2} \pi d u \\
& \quad \leq k_{5}\left(\left|x_{1}-x_{2}\right|^{2}+\left|y_{1}-y_{2}\right|^{2}\right) .
\end{aligned}
$$

Definition 2.1 The system (1) is said to be exponentially stable in the $p$ th moment if there exist a pair of positive constant $\delta$ and $K$ such that

$$
E|x(t)|^{p} \leq K E\|\psi\|^{p} e^{-\delta t}, \quad t \geq 0 .
$$

Lemma 2.2 ([2], Bukhölder-Davis-Gundy inequality) There exists a universal constant $J_{p}$ for any $p \in(0, \infty)$ such that for every continuous local martingale $M$ vanishing at zero and any stopping time $T$,

$$
E\left(\left|M_{T}\right|^{p}\right) \leq E\left(\sup _{0 \leq s \leq T}\left|M_{s}\right|^{p}\right) \leq J_{p} E\left(\langle M, M\rangle_{T}\right)^{\frac{p}{2}},
$$

where $\langle M, M\rangle$ is the cross-variation of $M$.

To study stability of the system (1), we rewrite (1) into

$$
\begin{aligned}
d\left[x(t)-G\left(t, x\left(t-\tau_{1}(t)\right)\right)\right]= & A\left(t, t-\tau_{2}(t)\right)\left(1-\tau_{2}^{\prime}(t)\right) g\left(x\left(t-\tau_{2}(t)\right)\right) d t \\
& +d\left(\int_{t-\tau_{2}(t)}^{t} A(t, s) g(x(s)) d s\right) \\
& +\sigma\left(t, x(t), x\left(t-\tau_{3}(t)\right)\right) d B(t) \\
& +\int_{R} h\left(t, x(t), x\left(t-\tau_{4}(t)\right), u\right) \tilde{N}(d t, d u),
\end{aligned}
$$

where

$$
A(t, s)=\int_{t}^{s} a(u, s) d u, \quad A\left(t, t-\tau_{2}(t)\right)=\int_{t}^{t-\tau_{2}(t)} a\left(u, t-\tau_{2}(t)\right) d u .
$$

\section{3 pth moment exponential stability}

In this section, we study the $p$ th moment exponential stability of (1).

Theorem 3.1 Suppose that there exists a continuous function $H(t):[0, \infty) \rightarrow R^{+}$such that it satisfies the following conditions:

(a) there exists a positive constant $n \geq 1$ such that $H(t)>n, t \in R^{+}$;

(b) For $t \in R^{+}, z \in[0, t]$ and a positive constant $\alpha<1$, the following inequality holds:

$$
\begin{aligned}
r:= & 3^{p-1}\left(2 k_{1}+k_{3} \int_{0}^{z} e^{-\int_{s}^{z} H(\mu) d \mu} A\left(s, s-\tau_{2}(s)\right)\left(1-\tau_{2}^{\prime}(s)\right) d s\right. \\
& \left.+k_{3} \int_{z-\tau_{2}(z)}^{z} A(t, v) d v+k_{3} \int_{0}^{z} e^{-\int_{s}^{z} H(\mu) d \mu} H(s) \int_{s-\tau_{2}(s)}^{s} A(s, v) d v d s\right)^{p} \\
& +4 \times 3^{p-1} k_{5}(1+\pi)\left(\int_{0}^{z} e^{-\int_{s}^{z} 2 H(\mu) d \mu} d s\right)^{\frac{p}{2}} \\
\leq & \alpha<1,
\end{aligned}
$$


where $p \geq 1$ and $A(t, v), A(s, s-\tau(s))$ are defined as (3). Then the zero solution of (1) shows pth moment exponential stability.

Proof Multiplying both sides of (2) by the factor $e^{\int_{0}^{t} H(\mu) d \mu}$, and integrating from 0 to any time $t<+\infty$, we obtain

$$
\begin{aligned}
x(t)= & {\left[\psi(0)-G\left(0, \psi\left(-\tau_{1}(0)\right)\right)\right] e^{-\int_{0}^{t} H(\mu) d \mu}+G\left(t, x\left(t-\tau_{1}(t)\right)\right) } \\
& +\int_{0}^{t} e^{-\int_{s}^{t} H(\mu) d \mu} H(s)\left[x(s)-G\left(s, x\left(s-\tau_{1}(s)\right)\right)\right] d s \\
& +\int_{0}^{t} e^{-\int_{s}^{t} H(\mu) d \mu} A\left(s, s-\tau_{2}(s)\right)\left(1-\tau_{2}^{\prime}(s)\right) g\left(x\left(s-\tau_{2}(s)\right)\right) d s \\
& +\int_{0}^{t} e^{-\int_{s}^{t} H(\mu) d \mu} d\left[\int_{s-\tau_{2}(s)}^{s} A(s, v) g(x(v)) d v\right] \\
& +\int_{0}^{t} e^{-\int_{s}^{t} H(\mu) d \mu} \sigma\left(s, x(s), x\left(s-\tau_{3}(s)\right)\right) d B(s) \\
& +\int_{0}^{t} e^{-\int_{s}^{t} H(\mu) d \mu} \int_{R} h\left(s, x(s), x\left(s-\tau_{4}(s)\right), u\right) \tilde{N}(d s, d u) .
\end{aligned}
$$

Performing an integration by parts, we obtain

$$
\begin{aligned}
x(t)= & {\left[\psi(0)-G\left(0, \psi\left(-\tau_{1}(0)\right)\right)\right] e^{-\int_{0}^{t} H(\mu) d \mu}+G\left(t, x\left(t-\tau_{1}(t)\right)\right) } \\
& +\int_{0}^{t} e^{-\int_{s}^{t} H(\mu) d \mu} H(s)\left[x(s)-G\left(s, x\left(s-\tau_{1}(s)\right)\right)\right] d s \\
& +\int_{0}^{t} e^{-\int_{s}^{t} H(\mu) d \mu} A\left(s, s-\tau_{2}(s)\right)\left(1-\tau_{2}^{\prime}(s)\right) g\left(x\left(s-\tau_{2}(s)\right)\right) d s \\
& +\int_{t-\tau_{2}(t)}^{t} A(t, v) g(x(v)) d v-e^{-\int_{0}^{t} H(\mu) d \mu} \int_{-\tau_{2}(0)}^{0} A(0, v) g(x(v)) d v \\
& -\int_{0}^{t} e^{-\int_{s}^{t} H(\mu) d \mu} H(s) \int_{s-\tau_{2}(s)}^{s} A(s, v) g(x(v)) d v d s \\
& +\int_{0}^{t} e^{-\int_{s}^{t} H(\mu) d \mu} \sigma\left(s, x(s), x\left(s-\tau_{3}(s)\right)\right) d B(s) \\
& +\int_{0}^{t} \int_{R} e^{-\int_{s}^{t} H(\mu) d \mu} h\left(s, x(s), x\left(s-\tau_{4}(s)\right), u\right) \tilde{N}(d s, d u) \\
:= & \sum_{i=1}^{9} I_{i}(t),
\end{aligned}
$$

where

$$
\begin{aligned}
& I_{1}(t)=\left[\psi(0)-G\left(0, \psi\left(-\tau_{1}(0)\right)\right)\right] e^{-\int_{0}^{t} H(\mu) d \mu}, \\
& I_{2}(t)=G\left(t, x\left(t-\tau_{1}(t)\right)\right), \\
& I_{3}(t)=\int_{0}^{t} e^{-\int_{s}^{t} H(\mu) d \mu} H(s)\left[x(s)-G\left(s, x\left(s-\tau_{1}(s)\right)\right)\right] d s, \\
& I_{4}(t)=\int_{0}^{t} e^{-\int_{s}^{t} H(\mu) d \mu} A\left(s, s-\tau_{2}(s)\right)\left(1-\tau_{2}^{\prime}(s)\right) g\left(x\left(s-\tau_{2}(s)\right)\right) d s,
\end{aligned}
$$




$$
\begin{aligned}
& I_{5}(t)=\int_{t-\tau_{2}(t)}^{t} A(t, v) g(x(v)) d v, \\
& I_{6}(t)=-e^{-\int_{0}^{t} H(\mu) d \mu} \int_{-\tau_{2}(0)}^{0} A(0, v) g(x(v)) d v, \\
& I_{7}(t)=-\int_{0}^{t} e^{-\int_{s}^{t} H(\mu) d \mu} H(s) \int_{s-\tau_{2}(s)}^{s} A(s, v) g(x(v)) d v d s, \\
& I_{8}(t)=\int_{0}^{t} e^{-\int_{s}^{t} H(\mu) d \mu} \sigma\left(s, x(s), x\left(s-\tau_{3}(s)\right)\right) d B(s), \\
& I_{9}(t)=\int_{0}^{t} \int_{R} e^{-\int_{s}^{t} H(\mu) d \mu} h\left(s, x(s), x\left(s-\tau_{4}(s)\right), u\right) \tilde{N}(d s, d u) .
\end{aligned}
$$

Let $\left(\mathcal{B},\|\cdot\|_{\mathcal{B}}\right)$ be the Banach space of all bounded and continuous in the $p$ th mean $\mathcal{F}_{0}$ adapted process $\phi(t, \omega):[-m(0), \infty) \times \Omega \rightarrow R$ with the following supremum norm:

$$
\|\phi\|_{\mathcal{B}}:=\sup _{t \geq 0} E|\phi(t)|^{p}, \quad \text { for } \phi \in \mathcal{B} .
$$

Moreover, let $Z$ denote the complete metric space with the supremum metric consisting of functions $\phi \in \mathcal{B}$ such that $\phi(s)=\psi(s)$ on $s \in[-m(0), 0]$ and $e^{\lambda t} E|\phi(t, \omega)|^{p} \rightarrow 0$ as $t \rightarrow \infty$, where $n>\lambda$. Then we define an operator $P: Z \rightarrow Z$ as follows:

$$
P(x)(t):= \begin{cases}\psi(t), & t \in[-m(0), 0] \\ \sum_{i=1}^{9} I_{i}(t), & t \geq 0 .\end{cases}
$$

We will finish this proof in three steps.

Step 1: we will show that the operator $P$ is continuous in the $p$ th moment on $[0, \infty)$. Let $x \in Z, t_{1} \geq 0$ and $|a|$ be sufficiently small. By using the $C_{p}$ inequality we obtain

$$
E\left|P\left(t_{1}+a\right)-P\left(t_{1}\right)\right|^{p} \leq 9^{p-1} \sum_{i=1}^{9} E\left|I_{i}\left(t_{1}+a\right)-I_{i}\left(t_{1}\right)\right|^{p} .
$$

As $|a| \rightarrow 0$, we have

$$
\begin{aligned}
& E\left|I_{1}\left(t_{1}+a\right)-I_{1}\left(t_{1}\right)\right|^{p} \\
& \quad=\left[\psi(0)-G\left(0, \psi\left(-\tau_{1}(0)\right)\right)\right] e^{-\int_{0}^{t_{1}+a} H(\mu) d \mu}-\left[\psi(0)-G\left(0, \psi\left(-\tau_{1}(0)\right)\right)\right] e^{-\int_{0}^{t_{1}} H(\mu) d \mu} \\
& \quad=\left[\psi(0)-G\left(0, \psi\left(-\tau_{1}(0)\right)\right)\right] e^{-\int_{0}^{t_{1}} H(\mu) d \mu}\left(e^{-\int_{t_{1}}^{t_{1}+a} H(\mu) d \mu}-1\right) \\
& \quad \rightarrow 0 \\
& \quad E\left|I_{2}\left(t_{1}+a\right)-I_{2}\left(t_{1}\right)\right|^{p} \\
& \quad=E\left|G\left(t_{1}+a, x\left(t_{1}+a-\tau_{1}\left(t_{1}+a\right)\right)\right)-G\left(t_{1}, x\left(t_{1}-\tau_{1}\left(t_{1}\right)\right)\right)\right|^{p} \\
& \quad \rightarrow 0, \\
& E\left|I_{3}\left(t_{1}+a\right)-I_{3}\left(t_{1}\right)\right|^{p} \\
& \quad=E \mid \int_{0}^{t_{1}+a} e^{-\int_{s}^{t_{1}+a} H(\mu) d \mu} H(s)\left[x(s)-G\left(s, x\left(s-\tau_{1}(s)\right)\right)\right] d s
\end{aligned}
$$


Yin et al. Advances in Difference Equations 2014, 2014:106

Page 6 of 14

http://www.advancesindifferenceequations.com/content/2014/1/106

$$
\begin{aligned}
& -\left.\int_{0}^{t_{1}} e^{-\int_{s}^{t_{1}} H(\mu) d \mu} H(s)\left[x(s)-G\left(s, x\left(s-\tau_{1}(s)\right)\right)\right] d s\right|^{p} \\
= & E \mid \int_{t_{1}}^{t_{1}+a} e^{-\int_{s}^{t_{1}+a} H(\mu) d \mu} H(s)\left[x(s)-G\left(s, x\left(s-\tau_{1}(s)\right)\right)\right] d s \\
& +\left.\int_{0}^{t_{1}} e^{-\int_{s}^{t_{1}} H(\mu) d \mu} H(s)\left(e^{-\int_{t_{1}}^{t_{1}+a} H(\mu) d \mu}-1\right)\left[x(s)-G\left(s, x\left(s-\tau_{1}(s)\right)\right)\right] d s\right|^{p} \\
\leq & 2^{p-1} E\left(\int_{t_{1}}^{t_{1}+a} e^{-\int_{s}^{t_{1}+a} H(\mu) d \mu}\left|H(s)\left[x(s)-G\left(s, x\left(s-\tau_{1}(s)\right)\right)\right]\right| d s\right)^{p} \\
& +2^{p-1} E\left(\int_{0}^{t_{1}} e^{-\int_{s}^{t_{1}} H(\mu) d \mu}\left(e^{-\int_{t_{1}}^{t_{1}+a} H(\mu) d \mu}-1\right)\right. \\
& \left.\times\left|H(s)\left[x(s)-G\left(s, x\left(s-\tau_{1}(s)\right)\right)\right]\right| d s\right)^{p} \\
\rightarrow & 0
\end{aligned}
$$

$$
\begin{aligned}
& E\left|I_{4}\left(t_{1}+a\right)-I_{4}\left(t_{1}\right)\right|^{p} \\
& =E \mid \int_{0}^{t_{1}+a} e^{-\int_{s}^{t_{1}+a} H(\mu) d \mu} A\left(s, s-\tau_{2}(s)\right)\left(1-\tau_{2}^{\prime}(s)\right) g\left(x\left(s-\tau_{2}(s)\right)\right) d s \\
& \quad-\left.\int_{0}^{t_{1}} e^{-\int_{s}^{t_{1}} H(\mu) d \mu} A\left(s, s-\tau_{2}(s)\right)\left(1-\tau_{2}^{\prime}(s)\right) g\left(x\left(s-\tau_{2}(s)\right)\right) d s\right|^{p} \\
& \leq 2^{p-1} E\left(\int_{t_{1}}^{t_{1}+a} e^{-\int_{s}^{t_{1}+a} H(\mu) d \mu}\left|A\left(s, s-\tau_{2}(s)\right)\left(1-\tau_{2}^{\prime}(s)\right) g\left(x\left(s-\tau_{2}(s)\right)\right)\right| d s\right)^{p} \\
& \quad+2^{p-1} E\left(\int_{0}^{t_{1}} e^{-\int_{s}^{t_{1}} H(\mu) d \mu}\left(e^{-\int_{t_{1}}^{t_{1}+a} H(\mu) d \mu}-1\right)\right. \\
& \left.\quad \times\left|A\left(s, s-\tau_{2}(s)\right)\left(1-\tau_{2}^{\prime}(s)\right) g\left(x\left(s-\tau_{2}(s)\right)\right)\right| d s\right)^{p} \\
& \rightarrow 0
\end{aligned}
$$

$$
\begin{array}{rl}
E\left|I_{5}\left(t_{1}+a\right)-I_{5}\left(t_{1}\right)\right|^{p} & A\left(t_{1}+a, v\right) g(x(v)) d v-\left.\int_{t_{1}-\tau_{2}\left(t_{1}\right)}^{t_{1}} A\left(t_{1}, v\right) g(x(v)) d v\right|^{p} \\
= & E \mid \int_{t_{1}+a-\tau_{2}\left(t_{1}+a\right)}^{t_{1}+a} t_{1}^{t_{1}+a} A\left(t_{1}+a, v\right) g(x(v)) d v \\
= & E \mid \int_{0}^{t_{1}}\left[A\left(t_{1}+a, v\right)-A\left(t_{1}, v\right)\right] g(x(v)) d v+\int_{t_{1}} \\
& +\int_{0}^{t_{1}-\tau_{2}\left(t_{1}\right)}\left[A\left(t_{1}, v\right)-A\left(t_{1}+a, v\right)\right] g(x(v)) d v \\
& -\left.\int_{t_{1}-\tau_{2}\left(t_{1}\right)}^{t_{1}+a-\tau_{2}\left(t_{1}+a\right)} A\left(t_{1}+a, v\right) g(x(v)) d v\right|^{p} \\
\leq & 4^{p-1}\left\{E\left[\int_{0}^{t_{1}}\left|A\left(t_{1}+a, v\right)-A\left(t_{1}, v\right)\right||g(x(v))| d v\right]^{p}\right. \\
& +E\left[\int_{t_{1}}^{t_{1}+a}\left|A\left(t_{1}+a, v\right) g(x(v))\right| d v\right]^{p} \\
& +E\left[\int_{0}^{t_{1}-\tau_{2}\left(t_{1}\right)}\left|A\left(t_{1}, v\right)-A\left(t_{1}+a, v\right)\right||g(x(v))| d v\right]^{p}
\end{array}
$$




$$
\left.+E\left[\int_{t_{1}-\tau_{2}\left(t_{1}\right)}^{t_{1}+a-\tau_{2}\left(t_{1}+a\right)}\left|A\left(t_{1}+a, v\right) g(x(v))\right| d v\right]^{p}\right\}
$$

$\rightarrow 0$,

$$
\begin{aligned}
E \mid & I_{6}\left(t_{1}+a\right)-\left.I_{6}\left(t_{1}\right)\right|^{p} \\
= & E\left|e^{-\int_{0}^{t_{1}+a} H(\mu) d \mu} \int_{-\tau_{2}(0)}^{0} A(0, v) g(\psi(v)) d v-e^{-\int_{0}^{t_{1}} H(\mu) d \mu} \int_{-\tau_{2}(0)}^{0} A(0, v) g(\psi(v)) d v\right|^{p} \\
= & E\left|e^{-\int_{0}^{t_{1}} H(\mu) d \mu}\left(e^{-\int_{t_{1}}^{t_{1}+a} H(\mu) d \mu}-1\right) \int_{-\tau_{2}(0)}^{0} A(0, v) g(x(v)) d v\right|^{p} \\
\rightarrow & 0 \\
E \mid & I_{7}\left(t_{1}+a\right)-\left.I_{7}\left(t_{1}\right)\right|^{p} \\
= & E \mid \int_{0}^{t_{1}+a} e^{-\int_{s}^{t_{1}+a} H(\mu) d \mu} H(s) \int_{s-\tau_{2}(s)}^{s} A(s, v) g(x(v)) d v d s \\
& -\left.\int_{0}^{t_{1}} e^{-\int_{s}^{t_{1}} H(\mu) d \mu} H(s) \int_{s-\tau_{2}(s)}^{s} A(s, v) g(x(v)) d v d s\right|^{p} \\
\leq & 2^{p-1}\left\{E\left[\int_{0}^{t_{1}} e^{-\int_{s}^{t_{1}} H(\mu) d \mu}\left|e^{-\int_{t_{1}}^{t_{1}+a} H(\mu) d \mu}-1\right| \int_{s-\tau_{2}(s)}^{s}|H(s) A(s, v) g(x(v))| d v d s\right]^{p}\right. \\
& \left.+E\left[\int_{t_{1}}^{t_{1}+a} e^{-\int_{s}^{t_{1}+a} H(\mu) d \mu} \int_{s-\tau_{2}(s)}^{s}|H(s) A(s, v) g(x(v))| d v d s\right]^{p}\right\} \\
\rightarrow & 0,
\end{aligned}
$$

$$
\begin{aligned}
E\left|I_{8}\left(t_{1}+a\right)-I_{8}\left(t_{1}\right)\right|^{p} & \\
= & E \mid \int_{0}^{t_{1}+a} e^{-\int_{s}^{t_{1}+a} H(\mu) d \mu} \sigma\left(s, x(s), x\left(s-\tau_{3}(s)\right)\right) d B(s) \\
& -\left.\int_{0}^{t_{1}} e^{-\int_{s}^{t_{1}} H(\mu) d \mu} \sigma\left(s, x(s), x\left(s-\tau_{3}(s)\right)\right) d B(s)\right|^{p} \\
\leq & 2^{p-1} E\left|\int_{0}^{t_{1}} e^{-\int_{s}^{t_{1}} H(\mu) d \mu}\left(e^{-\int_{t_{1}}^{t_{1}+a} H(\mu) d \mu}-1\right) \sigma\left(s, x(s), x\left(s-\tau_{3}(s)\right)\right) d B(s)\right|^{p} \\
& +2^{p-1} E\left|\int_{t_{1}}^{t_{1}+a} e^{-\int_{s}^{t_{1}+a} H(\mu) d \mu} \sigma\left(s, x(s), x\left(s-\tau_{3}(s)\right)\right) d B(s)\right|^{p} \\
\leq & 2^{p-1} J_{p} E\left[\int_{0}^{t_{1}} e^{-2 \int_{s}^{t} H(\mu) d \mu}\left(e^{-\int_{t_{1}}^{t_{1}+a} H(\mu) d \mu}-1\right)^{2} \sigma^{2}\left(s, x(s), x\left(s-\tau_{3}(s)\right)\right) d s\right]^{\frac{p}{2}} \\
& +2^{p-1} J_{p} E\left[\int_{t_{1}}^{t_{1}+a} e^{-2 \int_{s}^{t} H(\mu) d \mu} \sigma^{2}\left(s, x(s), x\left(s-\tau_{3}(s)\right)\right) d s\right]^{\frac{p}{2}} \\
\rightarrow & 0,
\end{aligned}
$$

$$
\begin{aligned}
& E\left|I_{9}\left(t_{1}+a\right)-I_{9}\left(t_{1}\right)\right|^{p} \\
& =E \mid \int_{0}^{t_{1}+a} \int_{R} e^{-\int_{s}^{t_{1}+a} H(\mu) d \mu} h\left(s, x(s), x\left(s-\tau_{4}(s)\right), u\right) \tilde{N}(d s, d u) \\
& \quad-\left.\int_{0}^{t_{1}} \int_{R} e^{-\int_{s}^{t_{1}} H(\mu) d \mu} h\left(s, x(s), x\left(s-\tau_{4}(s)\right), u\right) \tilde{N}(d s, d u)\right|^{p}
\end{aligned}
$$




$$
\begin{aligned}
& \leq 2^{p-1} E\left|\int_{0}^{t_{1}} \int_{R} e^{-\int_{s}^{t_{1}} H(\mu) d \mu}\left(e^{-\int_{t_{1}}^{t_{1}+a} H(\mu) d \mu}-1\right) h\left(s, x(s), x\left(s-\tau_{4}(s)\right), u\right) \tilde{N}(d s, d u)\right|^{p} \\
& \quad+2^{p-1} E\left|\int_{t_{1}}^{t_{1}+a} \int_{R} e^{-\int_{s}^{t_{1}+a} H(\mu) d \mu} h\left(s, x(s), x\left(s-\tau_{4}(s)\right), u\right) \tilde{N}(d s, d u)\right|^{p} \\
& \leq 2^{p-1} J_{p} E\left[\int_{0}^{t_{1}} \int_{R} e^{-2 \int_{s}^{t} H(\mu) d \mu}\left(e^{-\int_{t_{1}}^{t_{1}+a} H(\mu) d \mu}-1\right)^{2}\right. \\
& \left.\quad \times h^{2}\left(s, x(s), x\left(s-\tau_{4}(s)\right), u\right) \pi d s d u\right]^{\frac{p}{2}} \\
& \quad+2^{p-1} J_{p} E\left[\int_{t_{1}}^{t_{1}+a} \int_{R} e^{-2 \int_{s}^{t} H(\mu) d \mu} h^{2}\left(s, x(s), x\left(s-\tau_{4}(s)\right), u\right) \pi d s d u\right]^{\frac{p}{2}} \\
& \rightarrow 0 .
\end{aligned}
$$

Thus, we can conclude that the operator $P$ is $p$ th continuous on $[0, \infty)$.

Step 2: We will prove that $P(Z) \subseteq Z$. Then we only need to prove $e^{\lambda t} E\left|I_{i}(t)\right|^{p} \rightarrow 0$ as $t \rightarrow$ $\infty$ for $i=1, \ldots, 9$, where $n>\lambda$. In fact, for $\forall \varepsilon>0$, there exists $T>0$ such that $e^{\lambda t} E|x(t)|^{p}<\varepsilon$ and $e^{\lambda t} E\left|x\left(t-\tau_{j}(t)\right)\right|^{p}<\varepsilon, j=1,2,3$ when $t>T-m(0)$. We have

$$
\begin{aligned}
& e^{\lambda t} E\left|I_{1}(t)\right|^{p}= e^{\lambda t}\left|\psi(0)-G\left(0, \psi\left(-\tau_{1}(0)\right)\right)\right|^{p} e^{-p \int_{0}^{t} H(\mu) d \mu} \\
& \leq\left|\psi(0)-G\left(0, \psi\left(-\tau_{1}(0)\right)\right)\right|^{p} e^{-(p n-\lambda) t} \\
& \rightarrow 0, \\
& e^{\lambda t} E\left|I_{2}(t)\right|^{p}= e^{\lambda t} E\left|G\left(t, x\left(t-\tau_{1}(t)\right)\right)\right|^{p}=k_{1} e^{\lambda t} E\left|x\left(t-\tau_{1}(t)\right)\right|^{p} \leq k_{1} \varepsilon \rightarrow 0, \\
& e^{\lambda t} E\left|I_{3}(t)\right|^{p}= e^{\lambda t} E\left|\int_{0}^{t} e^{-\int_{s}^{t} H(\mu) d \mu} H(s)\left[x(s)-G\left(s, x\left(s-\tau_{1}(s)\right)\right)\right] d s\right|^{p} \\
& \leq e^{\lambda t} E\left[\int_{0}^{t} e^{-\int_{s}^{t} H(\mu) d \mu} H(s)\left(|x(s)|+k_{1}\left|x\left(s-\tau_{1}(s)\right)\right|\right) d s\right]^{p} \\
& \leq e^{\lambda t} E\left[\int_{0}^{t} e^{-\int_{s}^{t} H(\mu) d \mu} H(s)\left(|x(s)|+k_{1}\left|x\left(s-\tau_{1}(s)\right)\right|\right) d s\right] \\
& \leq e^{\lambda t} E\left[\left(\int_{0}^{t} e^{-\int_{s}^{t} H(\mu) d \mu} H(s) d s\right)^{p-1}\right. \\
&\left.\times \int_{0}^{t} e^{-\int_{s}^{t} H(\mu) d \mu} H(s)\left(|x(s)|+k_{1}\left|x\left(s-\tau_{1}(s)\right)\right|\right)^{p} d s\right] \\
& \leq e^{\lambda t} E\left[\int_{0}^{t} e^{-\int_{s}^{t} H(\mu) d \mu} H(s)\left(|x(s)|+k_{1}\left|x\left(s-\tau_{1}(s)\right)\right|\right)^{p} d s\right] \\
&= e^{\lambda t} E\left[\int_{0}^{T} e^{-\int_{s}^{t} H(\mu) d \mu} H(s)\left(|x(s)|+k_{1}\left|x\left(s-\tau_{1}(s)\right)\right|\right)^{p} d s\right] \\
&+e^{\lambda t} E\left[\int_{T}^{t} e^{-\int_{s}^{t} H(\mu) d \mu} H(s)\left(|x(s)|+k_{1}\left|x\left(s-\tau_{1}(s)\right)\right|\right)^{p} d s\right] \\
& \leq\left(1+k_{1}\right)^{p} E\left[e_{s \in[-m(0), T]}^{\lambda t} \int_{T}^{t} E\left[2^{p-1}\left(|x(s)|^{p}+k_{1}^{p}\left|x\left(s-\tau_{1}(s)\right)\right|^{p}\right)\right] e^{-\int_{s}^{t} H(\mu) d \mu} H(s) d s\right. \\
& s_{0}^{p} e^{-(n-\lambda) t+s n} d s \\
& \\
&
\end{aligned}
$$




$$
\begin{aligned}
& \leq\left(1+k_{1}\right)^{p} E\left[\sup _{s \in[-m(0), T]}|x(s)|^{p}\right] \int_{0}^{T} e^{-(n-\lambda) t+s n} \\
& +2^{p-1}\left(1+k_{1}^{p}\right) \varepsilon\left(1-e^{-\int_{T}^{t} H(\mu) d \mu}\right) \\
& \rightarrow 0 \text {, } \\
& e^{\lambda t} E\left|I_{4}(t)\right|^{p}=e^{\lambda t} E\left|\int_{0}^{t} e^{-\int_{s}^{t} H(\mu) d \mu} A\left(s, s-\tau_{2}(s)\right)\left(1-\tau_{2}^{\prime}(s)\right) g\left(x\left(s-\tau_{2}(s)\right)\right) d s\right|^{p} \\
& \leq e^{\lambda t} E\left[\int_{0}^{t} e^{-\int_{s}^{t} H(\mu) d \mu} k_{2} k_{3}\left|m(0)\left(1-\tau_{2}^{\prime}(s)\right) x\left(s-\tau_{2}(s)\right)\right| d s\right]^{p} \\
& \leq e^{\lambda t} E\left[\left(\int_{0}^{t} e^{-\int_{s}^{t} H(\mu) d \mu} d s\right)^{p-1}\right. \\
& \left.\times \int_{0}^{t} e^{-\int_{s}^{t} H(\mu) d \mu}\left(k_{2} k_{3}\right)^{p}\left|m(0)\left(1-\tau_{2}^{\prime}(s)\right) x\left(s-\tau_{2}(s)\right)\right|^{p} d s\right] \\
& \leq e^{\lambda t} E\left[\left(\int_{0}^{t} e^{-\int_{s}^{t} H(\mu) d \mu} H(s) d s\right)^{p-1}\right. \\
& \left.\times \int_{0}^{t} e^{-\int_{s}^{t} H(\mu) d \mu}\left(k_{2} k_{3}\right)^{p}\left|m(0)\left(1-\tau_{2}^{\prime}(s)\right) x\left(s-\tau_{2}(s)\right)\right|^{p} d s\right] \\
& \leq\left[k_{2} k_{3} m(0)\left(1+k_{4}\right)\right]^{p} e^{\lambda t} E\left[\int_{0}^{t} e^{-\int_{s}^{t} H(\mu) d \mu}\left|x\left(s-\tau_{2}(s)\right)\right|^{p} d s\right] \\
& \leq\left[k_{2} k_{3} m(0)\left(1+k_{4}\right)\right]^{p} e^{\lambda t} E\left[\int_{0}^{T} e^{-\int_{s}^{t} H(\mu) d \mu}\left|x\left(s-\tau_{2}(s)\right)\right|^{p} d s\right. \\
& \left.+\int_{T}^{t} e^{-\int_{s}^{t} H(\mu) d \mu} H(s)\left|x\left(s-\tau_{2}(s)\right)\right|^{p} d s\right] \\
& \leq\left[k_{2} k_{3} m(0)\left(1+k_{4}\right)\right]^{p}\left\{E\left[\sup _{s \in[-m(0), T]}|x(s)|^{p}\right] \int_{0}^{T} e^{-(n-\lambda) t+n s} d s\right. \\
& \left.+\varepsilon\left(1-e^{-\int_{T}^{t} H(\mu) d \mu}\right)\right\} \\
& \rightarrow 0 \text {, } \\
& e^{\lambda t} E\left|I_{5}(t)\right|^{p}=e^{\lambda t} E\left|\int_{t-\tau_{2}(t)}^{t} A(t, v) g(x(v)) d v\right|^{p} \\
& \leq e^{\lambda t} E\left[\int_{t-\tau_{2}(t)}^{t}|A(t, v) g(x(v))| d v\right]^{p} \\
& \leq e^{\lambda t} E\left[\left(\int_{t-m(0)}^{t}|A(t, v)|^{\frac{p}{p-1}} d v\right)^{p-1} \times \int_{t-m(0)}^{t}|g(x(v))|^{p} d v\right] \\
& \leq k_{2}^{p} k_{3} m^{2 p}(0) \varepsilon \rightarrow 0, \\
& e^{\lambda t} E\left|I_{6}(t)\right|^{p}=e^{\lambda t} e^{-p \int_{0}^{t} H(\mu) d \mu}\left|\int_{-\tau_{2}(0)}^{0} A(0, v) g(\psi(v)) d v\right|^{p} \\
& \leq e^{\lambda t} e^{-p \int_{0}^{t} H(\mu) d \mu}\left(\int_{-\tau_{2}(0)}^{0}|A(0, v) g(\psi(v))| d v\right)^{p} \\
& \leq\left[k_{2} k_{3} m^{2}(0) \max _{s \in[-m(0), 0]}|\psi(s)|\right]^{p} e^{-(n p-\lambda) t} \\
& \rightarrow 0 \text {, }
\end{aligned}
$$


Yin et al. Advances in Difference Equations 2014, 2014:106

Page 10 of 14

http://www.advancesindifferenceequations.com/content/2014/1/106

$$
\begin{aligned}
& e^{\lambda t} E\left|I_{7}(t)\right|^{p}=e^{\lambda t} E\left|\int_{0}^{t} e^{-\int_{s}^{t} H(\mu) d \mu} H(s) \int_{s-\tau_{2}(s)}^{s} A(s, v) g(x(v)) d v d s\right|^{p} \\
& \leq e^{\lambda t} E\left[\int_{0}^{t} e^{-\int_{s}^{t} H(\mu) d \mu} H(s) \int_{s-\tau_{2}(s)}^{s}|A(s, v) g(x(v))| d v d s\right]^{p} \\
& =e^{\lambda t} E\left[\int_{0}^{t} e^{-\int_{s}^{t} \frac{p-1}{p} H(\mu) d \mu} H^{\frac{p-1}{p}}(s)\right. \\
& \left.\times e^{-\int_{s}^{t} \frac{1}{p} H(\mu) d \mu} H^{\frac{1}{p}}(s) \int_{s-\tau_{2}(s)}^{s}|A(s, v) g(x(v))| d \nu d s\right]^{p} \\
& \leq e^{\lambda t} E\left[\left(\int_{0}^{t} e^{-\int_{s}^{t} H(\mu) d \mu} H(s)\right)^{p-1}\right. \\
& \left.\times \int_{0}^{t} e^{-\int_{s}^{t} H(\mu) d \mu} H(s)\left(\int_{s-\tau_{2}(s)}^{s}|A(s, v) g(x(v))| d v\right)^{p} d s\right] \\
& \leq e^{\lambda t} E\left[\int_{0}^{t} e^{-\int_{s}^{t} H(\mu) d \mu} H(s)\left(\int_{s-\tau_{2}(s)}^{s}|A(s, v) g(x(v))| d v\right)^{p} d s\right] \\
& \leq m^{p-1}(0) e^{\lambda t} E\left[\int_{0}^{t} e^{-\int_{s}^{t} H(\mu) d \mu} H(s) \int_{s-\tau_{2}(s)}^{s}|A(s, v) g(x(v))|^{p} d v d s\right] \\
& \leq k_{2}^{p} k_{3}^{p} m^{2 p-1}(0) e^{\lambda t} E\left[\int_{0}^{t} e^{-\int_{s}^{t} H(\mu) d \mu} H(s) \int_{s-\tau_{2}(s)}^{s}|x(v)|^{p} d v d s\right] \\
& \leq k_{2}^{p} k_{3}^{p} m^{2 p-1}(0) e^{\lambda t} E\left[\int_{0}^{T} e^{-\int_{s}^{t} H(\mu) d \mu} H(s) \int_{s-\tau_{2}(s)}^{s}|x(v)|^{p} d v d s\right. \\
& \left.+\int_{T}^{t} e^{-\int_{s}^{t} H(\mu) d \mu} H(s) \int_{s-\tau_{2}(s)}^{s}|x(v)|^{p} d v d s\right] \\
& \leq k_{2}^{p} k_{3}^{p} m^{2 p}(0)\left[E\left(\sup _{s \in[-m(0), T]}|x(s)|^{p}\right) e^{-(n-\lambda) t} \int_{0}^{T} e^{s n} H(s) d s\right. \\
& \left.+\varepsilon\left(1-e^{-\int_{T}^{t} H(\mu) d \mu}\right)\right] \\
& \rightarrow 0 \text {, } \\
& e^{\lambda t} E\left|I_{8}(t)\right|^{p}=e^{\lambda t} E\left|\int_{0}^{t} e^{-\int_{s}^{t} H(\mu) d \mu} \sigma\left(s, x(s), x\left(s-\tau_{3}(s)\right)\right) d B(s)\right|^{p} \\
& \leq J_{p} e^{\lambda t} E\left|\int_{0}^{t} e^{-2 \int_{s}^{t} H(\mu) d \mu} \sigma^{2}\left(s, x(s), x\left(s-\tau_{3}(s)\right)\right) d s\right|^{\frac{p}{2}} \\
& \leq k_{5} J_{p} e^{\lambda t} E\left[\int_{0}^{t} e^{-2 \int_{s}^{t} H(\mu) d \mu}\left(|x(s)|^{2}+\left|x\left(s-\tau_{3}(s)\right)\right|^{2}\right) d s\right]^{\frac{p}{2}} \\
& \leq k_{5} J_{p} e^{\lambda t} E\left[\int_{0}^{t} e^{-2 \frac{p-2}{p} \int_{s}^{t} H(\mu) d \mu} H(s)\right. \\
& \left.\times e^{-2 \frac{2}{p} \int_{s}^{t} H(\mu) d \mu}\left(|x(s)|^{2}+\left|x\left(s-\tau_{3}(s)\right)\right|^{2}\right) d s\right]^{\frac{p}{2}} \\
& \leq k_{5} J_{p} e^{\lambda t} E\left[\left(\int_{0}^{t} e^{-2 \int_{s}^{t} H(\mu) d \mu} H(s) d s\right)^{\frac{p-2}{2}}\right. \\
& \left.\times \int_{0}^{t} e^{-2 \int_{s}^{t} H(\mu) d \mu}\left(|x(s)|^{2}+\left|x\left(s-\tau_{3}(s)\right)\right|^{2}\right)^{\frac{p}{2}} d s\right]
\end{aligned}
$$




$$
\begin{aligned}
\leq & k_{5} J_{p} e^{\lambda t} E\left[\int_{0}^{t} e^{-2 \int_{s}^{t} H(\mu) d \mu}\left(|x(s)|^{2}+\left|x\left(s-\tau_{3}(s)\right)\right|^{2}\right)^{\frac{p}{2}} d s\right] \\
\leq & 2^{\frac{p}{2}-1} k_{5} e^{\lambda t} E\left[\int_{0}^{t} e^{-2 \int_{s}^{t} H(\mu) d \mu}\left(|x(s)|^{p}+\left|x\left(s-\tau_{3}(s)\right)\right|^{p}\right) d s\right] \\
\leq & 2^{\frac{p}{2}-1} k_{5} J_{p} e^{\lambda t} E\left[\int_{0}^{T} e^{-2 \int_{s}^{t} H(\mu) d \mu}\left(|x(s)|^{p}+\left|x\left(s-\tau_{3}(s)\right)\right|^{p}\right) d s\right. \\
& \left.+\int_{T}^{t} e^{-2 \int_{s}^{t} H(\mu) d \mu} H(s)\left(|x(s)|^{p}+\left|x\left(s-\tau_{3}(s)\right)\right|^{p}\right) d s\right] \\
\leq & 2^{\frac{p}{2}-1} k_{5} J_{p}\left[2 E\left(\sup _{s \in[-m(0), T]}|x(s)|^{p}\right) \int_{0}^{T} e^{-(2 n-\lambda) t+2 n s} d s\right. \\
& +\varepsilon\left(1-e^{\left.-2 \int_{T}^{t} H(\mu) d \mu\right)}\right] \\
\rightarrow & 0 .
\end{aligned}
$$

By the same method as in the inequality of $e^{\lambda t} E\left|I_{8}(t)\right|^{p}$, we get the following inequality:

$$
\begin{aligned}
e^{\lambda t} E\left|I_{9}(t)\right|^{p} & =e^{\lambda t} E\left|\int_{0}^{t} \int_{R} e^{-\int_{s}^{t} H(\mu) d \mu} h\left(s, x(s), x\left(s-\tau_{4}(s)\right), u\right) \tilde{N}(d s, d u)\right|^{p} \\
& \leq\left.\left. J_{p} e^{\lambda t} E\left|\int_{0}^{t} \int_{R} e^{-2 \int_{s}^{t} H(\mu) d \mu}\right| h\left(s, x(s), x\left(s-\tau_{4}(s)\right), u\right)\right|^{p} \pi(d u) d s\right|^{\frac{p}{2}} \\
& \leq k_{5} J_{p} \pi e^{\lambda t} E\left[\int_{0}^{t} e^{-2 \int_{s}^{t} H(\mu) d \mu}\left(|x(s)|^{2}+\left|x\left(s-\tau_{3}(s)\right)\right|^{2}\right) d s\right]^{\frac{p}{2}} \\
& \rightarrow 0 .
\end{aligned}
$$

From the above inequalities, we can conclude that the operator $P$ maps $Z$ into itself.

Step 3: Finally, we will prove that the operator $P$ is contractive. For any $x, y \in Z$, we have

$$
\begin{aligned}
E \sup _{z \in[0, t]}|P(x)(z)-P(y)(z)|^{p} \\
=E \sup _{z \in[0, t]} \mid G\left(t, x\left(t-\tau_{1}(t)\right)\right)-G\left(t, y\left(t-\tau_{1}(t)\right)\right) \\
\quad+\int_{0}^{t} e^{-\int_{s}^{t} H(\mu) d \mu} H(s)\left[x(s)-G\left(s, x\left(s-\tau_{1}(s)\right)\right)-y(s)+G\left(s, y\left(s-\tau_{1}(s)\right)\right)\right] d s \\
+\int_{0}^{t} e^{-\int_{s}^{t} H(\mu) d \mu} A\left(s, s-\tau_{2}(s)\right)\left(1-\tau_{2}^{\prime}(s)\right)\left[g\left(x\left(s-\tau_{2}(s)\right)\right)\right. \\
\left.\quad-g\left(y\left(s-\tau_{2}(s)\right)\right)\right] d s+\int_{t-\tau_{2}(t)}^{t} A(t, v)[g(x(v))-g(y(v))] d v \\
\quad-\int_{0}^{t} e^{-\int_{s}^{t} H(\mu) d \mu} H(s) \int_{s-\tau_{2}(s)}^{s} A(s, v)[g(x(v))-g(y(v)) d v] d s \\
\quad+\int_{0}^{t} e^{-\int_{s}^{t} H(\mu) d \mu}\left[\sigma\left(s, x(s), x\left(s-\tau_{3}(s)\right)\right)-\sigma\left(s, y(s), y\left(s-\tau_{3}(s)\right)\right)\right] d B(s) \\
\quad+\int_{0}^{t} \int_{R} e^{-\int_{s}^{t} H(\mu) d \mu}\left[h\left(s, x(s), x\left(s-\tau_{4}(s)\right), u\right)\right.
\end{aligned}
$$




$$
\begin{aligned}
& \left.-h\left(s, y(s), y\left(s-\tau_{4}(s)\right), u\right)\right]\left.\tilde{N}(d s, d u)\right|^{p} \\
\leq & E \sup _{z \in[0, t]}|x(z)-y(z)|^{p} \sup _{z \in[0, t]}\left\{3 ^ { p - 1 } \left(2 k_{1}\right.\right. \\
& +k_{3} \int_{0}^{z} e^{-\int_{s}^{z} H(\mu) d \mu} A\left(s, s-\tau_{2}(s)\right)\left(1-\tau_{2}^{\prime}(s)\right) d s \\
& \left.+k_{3} \int_{z-\tau_{2}(z)}^{z} A(t, v) d v+k_{3} \int_{0}^{z} e^{-\int_{s}^{z} H(\mu) d \mu} H(s) \int_{s-\tau_{2}(s)}^{s} A(s, v) d v d s\right)^{p} \\
& \left.+4 \times 3^{p-1} k_{5}(1+\pi)\left(\int_{0}^{z} e^{-\int_{s}^{z} 2 H(\mu) d \mu} d s\right)^{\frac{p}{2}}\right\} \\
\leq & \alpha<1 .
\end{aligned}
$$

Thus $P$ is a contraction mapping.

\section{Remarks and an example}

Remark 4.1 If $G\left(t, x\left(t-\tau_{1}(t)\right)\right)=0, \tau_{2}(t)=L, a(t, s)=p(s-t), \sigma\left(t, x(t), x\left(t-\tau_{3}(t)\right)\right)=\sigma(t)$ and $h\left(t, x(t), x\left(t-\tau_{1}(t)\right), u\right)=0$, then the system (1) turns into

$$
d x(t)=-\left(\int_{t-L}^{t} p(s-t) g(x(s)) d s\right) d t+\sigma(t) d B(t), \quad t \geq 0,
$$

which was recently studied in [14] by second moment, i.e., $p=2$.

Remark 4.2 When $G\left(t, x\left(t-\tau_{1}(t)\right)\right)=0, \tau_{2}(t)=\tau, a(t, s)=q(s-t), g(x(s))=f(x(s))$, $\sigma\left(t, x(t), x\left(t-\tau_{3}(t)\right)\right)=g(t)$ and $\int_{R} h\left(t, x(t), x\left(t-\tau_{1}(t)\right), u\right) d u=h(t)$, the system (1) degrades into

$$
d x(t)=-\left(\int_{t-L}^{t} q(s-t) f(x(s)) d s\right) d t+g(t) d B(t)+h(t) d \tilde{N}(t), \quad t \geq 0 .
$$

This system has been investigated in [15]. However, our model (1) is more general.

Example 4.1 We consider the following stochastic differential equation with variable delays:

$$
\begin{aligned}
d\left[x(t)-a_{1} x\left(t-\tau_{1}(t)\right)\right]= & -a_{2}\left(\int_{t-\tau_{2}(t)}^{t} x(s) d s\right) d t+a_{3} x\left(t-\tau_{3}(t)\right) d B(t) \\
& +a_{4} x\left(t-\tau_{4}(t)\right) d \tilde{N}(t), \quad t \geq 0 .
\end{aligned}
$$

Its initial condition is equipped with $x(0)=c$ (constant) and $a_{i}, i=1,2,3,4$, are positive constants. $\tau_{1}(t), \tau_{3}(t)$ and $\tau_{4}(t)$ are variable delays. Set $\tau_{2}(t) \equiv 1$ and $H(t) \equiv 4$, then according to the definition of $r$ under the condition (b) of Theorem 3.1, for $t \in R^{+}, z \in[0, t]$ we can calculate and obtain

$$
\begin{aligned}
r= & 3^{p-1}\left(2 a_{1}+a_{2} \int_{z-1}^{z}(v-t) d v+\int_{0}^{z} e^{-\int_{s}^{z} 4 d \mu} 4 \int_{s-1}^{s} a_{2}(v-s) d v d s\right)^{p} \\
& +4 \times 3^{p-1} \max \left\{a_{3}, a_{4}\right\}(1+\pi)\left(\int_{0}^{z} e^{-\int_{s}^{z} 8 d \mu} d s\right)^{\frac{p}{2}}
\end{aligned}
$$




$$
\begin{aligned}
= & 3^{p-1}\left(2 a_{1}-a_{2}(t-z+0.5)-0.5 a_{2}\left(1-e^{-4 z}\right)\right)^{p} \\
& +\frac{3^{p-1}}{2} \max \left\{a_{3}, a_{4}\right\}(1+\pi)\left(1-e^{-8 z}\right)^{\frac{p}{2}} \\
\leq & 3^{p-1}\left(2 a_{1}-0.5 a_{2}\right)^{p}+\frac{3^{p-1}}{2} \max \left\{a_{3}, a_{4}\right\}(1+\pi) .
\end{aligned}
$$

Let $p=1, a_{1}=0.1, a_{2}=0.2, a_{3}=a_{4}=0.04$, then by (8) we have $r \leq 0.2$. As a result, according to Theorem 3.1 we can conclude that the zero solution of (7) is one of first moment exponential stability.

\section{Conclusion}

In this paper, we have investigated the stochastic neutral Volterra-Levin equation with Lévy noise and variable delays, and we obtained the condition of $p$ th moment exponential stability by fixed point theory and the Bukhölder-Davis-Gundy inequality. Presently known results are improved and generalized. However, there are many problems to be solved for the stochastic Volterra-Levin equation, such as stability of this equation driven by factional Brownian motion [18], persistence, and so on.

\section{Competing interests}

The authors declare that they have no competing interests.

\section{Authors' contributions}

HY carried out the main parts of the draft. XX and XW provided the main idea of this paper. SX gave many valuable suggestions on how to modify this paper and responded the reviewers' comments. All authors checked and approved the final manuscript.

\section{Acknowledgements}

The authors would like to thank the anonymous referees for their carefulness, helpful comments and suggestions which can greatly improve this paper. This work was partially supported by the NSFC (Nos. 61304161 and 11301260), the NSF of Jiangxi (Nos. 20132BAB211003, 20132BAB211037) and the YFED of Jiangxi (No. GJJ13078) of China, the Education and Reform of Jiangxi (No. JXJG-13-1-3)

Received: 26 December 2013 Accepted: 25 March 2014 Published: 08 Apr 2014

\section{References}

1. Mao, X: Stochastic Differential Equations and Their Applications. Horwood, Chichester (1997)

2. Mao, X: Exponential Stability of Stochastic Differential Equations. Marcel Dekker, New York (1994)

3. Luo, J: Stability of stochastic partial differential equations with infinite delays. J. Comput. Appl. Math. 222(2), 364-371 (2008)

4. Sakthivel, R, Luo, J: Asymptotic stability of impulsive stochastic partial differential equations with infinite delays. J. Math. Anal. Appl. 356(1), 1-6 (2009)

5. Ren, Y, Sakthivel, R: Existence, uniqueness, and stability of mild solutions for second-order neutral stochastic evolution equations with infinite delay and Poisson jumps. J. Math. Phys. 53(7), 073517 (2012)

6. Sakthivel, R, Ren, Y: Exponential stability of second-order stochastic evolution equations with Poisson jumps. Commun. Nonlinear Sci. Numer. Simul. 17(12), 4517-4523 (2012)

7. Sakthivel, R, Ren, Y, Kim, H: Asymptotic stability of second-order neutral stochastic differential equations. J. Math. Phys. 51(5), 052701 (2010)

8. Burton, TA: Perron-type stability theorems for neutral equations. Nonlinear Anal., Theory Methods Appl. 55(3), 285-297 (2003)

9. Burton, TA, Zhang, B: Fixed points and stability of an integral equation: nonuniqueness. Appl. Math. Lett. 17(7), 839-846 (2004)

10. Burton, TA: Fixed points and stability of a nonconvolution equation. Proc. Am. Math. Soc. 132, 3679-3687 (2004)

11. Burton, TA: Stability by fixed point theory or Liapunov theory: a comparison. Fixed Point Theory 4(1), 15-32 (2003)

12. Levin, JJ: The asymptotic behavior of the solution of a Volterra equation. Proc. Am. Math. Soc. 14(4), $534-541$ (1963)

13. Zhao, D, Yuan, S: 3/2-stability conditions for a class of Volterra-Levin equations. Nonlinear Anal., Theory Methods Appl. 94, 1-11 (2014)

14. Luo, J: Fixed points and exponential stability for stochastic Volterra-Levin equations. J. Comput. Appl. Math. 234(3), 934-940 (2010)

15. Guo, L, Zhu, Q: Stability analysis for stochastic Volterra-Levin equations with Poisson jumps: fixed point approach. J. Math. Phys. 52(4), 042702 (2011)

16. Jin, C, Luo, J: Stability of an integro-differential equation. Comput. Math. Appl. 57(7), 1080-1088 (2009) 
17. Ardjouni, A, Djoudi, A: Stability in nonlinear neutral integro-differential equations with variable delay using fixed point theory. J. Appl. Math. Comput. 44, 317-336 (2013)

18. Biagini, F: Stochastic Calculus for Fractional Brownian Motion and Applications. Springer, London (2008)

10.1186/1687-1847-2014-106

Cite this article as: Yin et al.: pth moment stability in stochastic neutral Volterra-Levin equation with Lévy noise and variable delays. Advances in Difference Equations 2014, 2014:106

Submit your manuscript to a SpringerOpen ${ }^{\circ}$ journal and benefit from:

- Convenient online submission

- Rigorous peer review

- Immediate publication on acceptance

- Open access: articles freely available online

- High visibility within the field

- Retaining the copyright to your article

Submit your next manuscript at $\boldsymbol{~ s p r i n g e r o p e n . c o m ~}$ 